\title{
II. RADIOAGTIVITÉ
}

\section{LES NIVEAUX EXCITÉS DES NOYAUX DÉFORMÉS}

\author{
Par N. MARTY, \\ Laboratoire de Physique et Chimie Nucléaires du Collège de France.
}

\begin{abstract}
Sommaire. - Rappel des conditions d'existence et des propriétés (énergie-spin) des niveaux excités des noyaux attribuables à des mouvements collectifs de rotation :

a) noyaux pair pair ;

b) noyaux impairs.

Influence des niveaux excités de particules d'énergie peu élevée. Existence de transitions $\mathrm{E}_{1}$ émises de niveaux faiblement excités. Interprétation dans le cas des noyaux impairs, de ces transitions $\mathrm{E}_{1}$ ainsi que des propriétés des autres états excités par les modifications des niveaux de particules dans les noyaux fortement déformés. Exemple schémas de ${ }^{175} \mathrm{Lu}$ et de ${ }^{153} \mathrm{Eu}$.
\end{abstract}

Abstract. - Short review of the conditions of existence and properties (energy and spin) of the excited levels due to rotational motion :

a) even even nuclei ;

b) odd nuclei.

Structure of particle levels in deformed nuclei, evidence of $\mathrm{E}_{1}$ transitions.

On connaissait avec précision, depuis les premières années de la radioactivité, les énergies de certaines transitions $\gamma$, fortement converties, accompagnant la désintégration de corps radioactifs naturels. Le développement et l'apparition de techniques nouvelles a permis dans ces dix dernières années une étude beaucoup plus détaillée des schémas de niveaux des noyaux formés à partir d'isotopes radioactifs : perfectionnement de la spectrométrie $\alpha$ et $\beta$, mise au point de la spectrométrie $\gamma$ à l'aide des compteurs à scintillations et des compteurs proportionnels ; enfin développement des méthodes de coïncidences, et en particulier des coincidences rapides qui permettent de mesurer des périodes de l'ordre de $10^{-10} \mathrm{~s}$, et adaptation de ces méthodes aux mesures de corrélations angulaires.

Parallèlement le calcul des coefficients de conversion dans les couches $K$ et $L$, calcul qui se poursuit encore actuellement, a permis de déterminer avec certitude la nature des rayonnements d'après leurs coefficients de conversion.

Un grand nombre des résultats expérimentaux ainsi rassemblés pouvait s'expliquer d'après l'hypothèse du modèle en couche de JensenMayer [1], [2] surtout dans le cas des noyaux de numéro atomique $Z$ faible ou moyen, ou au voisinage des couches saturées.

Il restait cependant tout un domaine où il n'était pas possible d'interpréter de façon satisfaisante le nombre sans cesse croissant de données expérimentales : celui des noyaux qui ont un nombre de protons et de neutrons s'écartant fortement des nombres magiques ; un premier groupe est constitué par les noyaux de numéro atomique compris entre 60 et 80 , leur nombre de neutrons étant compris entre 88 et 120 ; le deuxième groupe est constitué par les noyaux très lourds : $Z>88$ et $N>136$.

Prenons par exemple deux noyaux ${ }^{100} \mathrm{Tm}^{169}$ et ${ }^{104} \mathrm{Lu}^{175}$ dont le nombre de protons diffère de deux : leurs spins mesurés sont respectivement $1 / 2$ et $7 / 2$. Klinkenberg [3] justifie ces valeurs en supposant que pour ${ }^{169} \mathrm{Tm}$, la couche $g_{7 / 2}$ est totalement remplie, les couches $d_{5 / 2}$ et $h_{11 / 2}$ sont partiellement occupées, un proton non couplé occupant la couche $s_{1 / 2}$ donne son spin au noyau ; pour ${ }^{175} \mathrm{Lu}$ au contraire le spin $7 / 2$, provient de ce que la couche $g_{7 / 2}$ a un nombre impair de particules. Pour les niveaux excités, les interprétations possibles sont encore plus nombreuses en raison $\mathrm{du}$ grand nombre de sous-couches d'énergie voisine.

C'est aussi dans ce domaine de nombre de masses que les noyaux présentent un moment quadrupolaire élevé (les moments quadrupolaires ont surtout été mesurés pour des noyaux du groupe 1). Ceci conduit à penser que les noyaux qui ont un grand nombre de neutrons et de protons en dehors des couches fermées sont très déformés ; on doit alors s'attendre à des mouvements d'ensemble de la masse nucléaire ; c'est ce qu'a considéré A. Bohr [4], [5], [6] dans sa théorie du modèle unifié. En première approximation les mouvements du noyau sont séparés en mouvements intrinsèques des nucléons par rapport au champ nucléaire lente- 
ment variable et en mouvements collectifs de rotation et de vibration; la fonction d'onde est alors du type $\psi=X_{\text {nucl }}$. $\varphi_{\text {vib }} . D_{\text {rot }}$.

Ceci est justifié dans l'hypothèse du couplage fort. Si l'on considère que les noyaux déformés ont un axe de symétrie, le vecteur moment angulaire total $\vec{j}$ des particules a un mouvement de précession rapide autour de l'axe de symétrie $\mathrm{z}^{\prime}$, sa projection $\Omega$ sur cet axe reste constante (voir fig. 1).

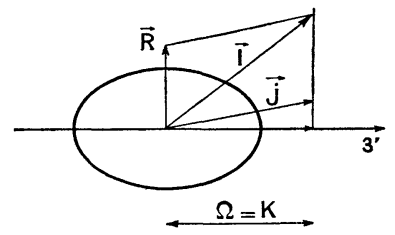

FIG. 1.

Les mouvements de rotation du noyau sont lents et préservent sa forme. Le moment angulaire nucléaire total $\vec{I}$ défini par ses projections $M$ sur un axe fixe et $K$ sur l'axe de symétrie du noyau, est la somme du moment angulaire $\stackrel{\leftarrow}{j}$ des particules et du moment angulaire collectif de rotation $\vec{R}$; celui-ci dans le cas de mouvements irrotationnels est perpendiculaire à l'axe de symétrie : on a $\Omega=K$ et à l'état fondamental où $R=0$, $\Omega=K=I$. Lorsque $R$ est différent de 0 , apparaissent les niveaux excités de rotation.

Dans les noyaux pair-pair l'énergie de ces états est donnée par

$$
E_{\text {rot }}=\frac{h^{2}}{2 \mathfrak{J}} I(I+1)
$$

où $\mathscr{J}$ est le moment d'inertie du noyau; le spin ne pouvant prendre que les valeurs paires $0,2,4,6$ : les - niveaux de rotation ont toujours la même parité que le niveau fondamental. On doit donc s'attendre à observer dans les noyaux pair-pair un premier niveau excité de spin 2 de faible énergie; des noyaux de déformation semblable auront des moments d'inertie voisins, les niveaux excités auront alors des énergies voisines; c'est effectivement ce que l'on observe, les noyaux du groupe 1 $(155<A<185)$ ont un premier niveau $2+$ d'énergie voisine de $100 \mathrm{keV}$; les noyaux très lourds du groupe 2 ont leur premier niveau excité de spin $2+$ d'énergie voisine de $60 \mathrm{keV}$. On trouve aussi dans un grand nombre de cas un deuxième état excité de spin 4, le rapport des énergies des niveaux $4+$ et $2+, E_{4} / E_{2}$ est bien de l'ordre de $\frac{10}{3}$. Ceci est d'autant plus remarquable que dès qu'on se rapproche de la région des couches saturées, l'énergie des premiers niveaux excités s'élève, le $2^{\mathrm{e}}$ niveau excité a souvent un spin $2+$ [7]. Par exemple alors que les noyaux ${ }_{62}^{90} \mathrm{Sm}^{152}$, ${ }_{64}^{90} \mathrm{Gd}^{154},{ }_{64}^{92} \mathrm{Gd}^{156}$ présentent bien un spectre de rotation pour leurs deux premiers états excités, le rapport $E_{4} / E_{2}$ pour ${ }_{62}^{88} \mathrm{Sm}^{150}$ et ${ }_{64}^{88} \mathrm{Gd}^{192}$ est 2,2 , il semble qu'il y ait une discontinuité des propriétés des noyaux lorsque le nombre de neutrons passe de 88 à 90 .

Enfin nous citerons l'exemple maintenant classique de ${ }_{72}^{180} \mathrm{Hf}$ où l'on observe un spectre de rotation avec des niveaux de spin $2+, 4+, 6+$, $8+;$ le léger écart entre les valeurs expérimentales de l'énergie des niveaux et les valeurs théoriques calculées d'après la formule (1) est dû à l'existence à des énergies de l'ordre de 2 à $3 \mathrm{MeV}$ de niveaux de vibration. L'interaction de ces mouvements de vibration avec les mouvements de rotation, plus importante lorsque ces derniers ne sont plus extrêmement lents donne lieu à un terme correctif négatif proportionnel à $-I^{2}(I+1)^{2}$. L'accord entre les valeurs expérimentales et les valeurs calculées, compte tenu de ce terme correctif, est très bon.

Dans les noyaux de $A$ impair, la théorie des niveaux de rotation prévoit des niveaux excités de $\operatorname{spin} I=I_{0}, I_{0}+1, I_{0}+2$ où $I_{0}$ est le spin du niveau fondamental; leur énergie étant

$$
E_{\text {rot }}=\frac{h^{2}}{2 y}\left[I(I+1)-I_{0}\left(I_{0}+1\right)\right] .
$$

Dans le cas où $\Omega=1 / 2$, le moment orbital des particules peut avoir une composante nulle le long de l'axe de symétrie, le spin de la particule est alors partiellement découplé du mouvement de rotation ; l'expression de l'énergie des niveaux de rotation présente un terme supplémentaire :

$$
E_{\mathrm{rot}}=\frac{h^{2}}{2 J}\left[I(I+1)-a(-1)^{I+1 / 2}(I+1 / 2)\right]
$$

Ces prévisions ont été amplement vérifiées dans l'étude des niveaux excités obtenus par désintégration $\alpha, \beta$ et capture électronique et surtout par excitation coulombienne des noyaux dans le domaine de $Z$ compris entre 60 et 80 . Le schéma des niveaux de ${ }_{69}^{100} \mathrm{Tm}^{169}$ [8] donne un exemple des niveaux de rotation d'un noyau pour lequel $\Omega=1 / 2 ;$ les énergies des transitions des premiers et deuxième niveau ont permis de déterminer $\mathcal{J}$ et $a$; on en déduit alors les énergies des niveaux 3 et 4 en bon accord avec les valeurs expérimentales.

Jusqu'à présent nous n'avons considéré qu'une famille de niveaux de rotation; s'il existe des niveaux excités de particules ayant des énergies peu élevées, on doit s'attendre à une interaction entre les états de rotation des deux familles [9]; en fait il n'y a mélange important des états que pour les niveaux de même spin appartenant à deux bandes de moment angulaire total $K$ et $K+1$. Dans le schéma de niveaux de ${ }^{183} \mathrm{~W}$ [10] 
où l'on a pu montrer l'existence de deux bandes de rotation pour lesquelles $K=1 / 2$ et $3 / 2$ les valeurs expérimentales des énergies des niveaux sont plus élevées que celles calculées d'après la formule (3) ; on retrouve bien les valeurs expérimentales si l'on fait intervenir le terme correctif dû à l'interaction entre les deux bandes de rotation [9].

Cependant un certain nombre de faits ne peuvent s'expliquer par les considérations précédentes : On a mis en évidence dans plusieurs noyaux lourds de $Z$ et $A$ pairs ou de $A$ impair des transitions dipôle électrique $E_{1}$ émises de niveaux proches du fondamental. Nous avons représenté figure 2 les schémas

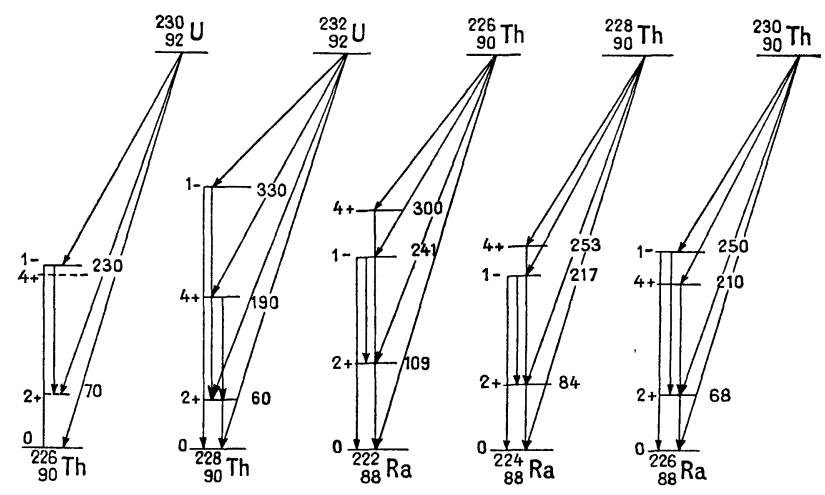

FIg. 2.

de niveaux des noyaux où apparaissent ces transitions [11], [12], [13]. La nature 1 - du niveau de départ a été établie par corrélations angulaires entre $\alpha$ et $\gamma$, d'après le coefficient de conversion des photons ainsi que d'après les intensités relatives des photons émis de ce niveau aux niveaux 0 et $2+$.
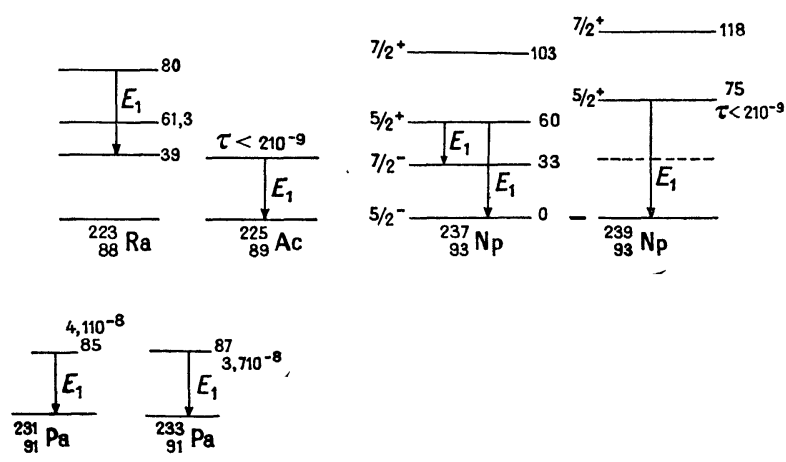

Fig. 3.

Des transitions semblables $E_{1}[14]$, [15], [16], [17], [18], [19] ont été trouvées dans les noyaux lourds de $A$ impair ; les mieux établies sont rassemblées figure 3 . Dans le cas de ${ }_{93}^{\mathbf{2 3 7}} \mathrm{Np}$ et ${ }_{9 \mathbf{9}}^{\mathbf{2 3 9}} \mathrm{Np}$, les désintégrations $\alpha$ importantes aboutissent aux niveaux de parité,$+ 5 / 2+, 7 / 2+$ et $9 / 2+$, niveaux de rotation de la famille $K=5 / 2$; les transitions au niveau fondamental de parité sont faibles. On peut aussi indiquer l'existence des transitions $E_{1}$ dans les noyaux ${ }_{91}^{231} \mathrm{~Pa}$ et ${ }_{91}^{233} \mathrm{~Pa}$; elles ont une période très longue (de l'ordre de $410^{-8}$ s) alors que pour les transitions $E_{1}$ de ${ }_{89}^{225} \mathrm{Ac}$ et ${ }_{93}^{239} \mathrm{~Np}$ les périodes des $E_{1}$ sont inférieures aे $210^{-9}{ }^{8 .}$.

D'autres transitions $E_{1}$ ont été mises en évidence pour des noyaux du groupe 1 ; nous en parlerons par la suite plus en détail.

Ces niveaux de spin peu différent et de parité opposée à ceux des niveaux avoisinants ne sont pas dus à des mouvements collectifs, ils s'expliquent difficilement si l'on se référe à la succession des niveaux prévue par le modèle en couche de Jensen-Mayer.

Rasmussen [20] a donné une première interprétation de ces transitions $E_{1}$. Si l'on admet un couplage fort entre les mouvements des particules individuelles et les déformations de surface du noyau, il peut y avoir des transitions entre états qui ont des fonctions d'ondes relatives aux particules extrêmement différentes et cependant des spins voisins.

Cette interprétation de Rasmussen s'est trouvée justifiée par les calculs complets réalisés par Nilsson [21]. Reprenant les hypothèses de base du modèle collectif unifié par A. Bohr suivant lesquelles on peut séparer, pour un noyau très déformé, les fonctions d'ondes relatives aux mouvements des particules des fonctions d'ondes relatives aux mouvements collectifs, Nilsson calcule les fonctions d'ondes des particules dans le champ correspondant à un noyau déformé - ce champ sera celui d'un noyau présentant un axe de symétrie et un plan de symétrie perpendiculaire à cet axe. Il choisit pour l'hamiltonien de la particule la forme suivante :

$$
H=H_{0}+C \bar{l} \bar{s}+D \bar{l}^{2}
$$

$H_{0}$ est lui-même séparable en deux termes, l'un présentant une symétrie sphérique, l'autre $H_{\delta}$, proportionnel au facteur $\delta$ de déformation, représente le couplage de la particule à l'axe de symétrie du noyau. Le terme en $\bar{\delta} \bar{s}$ est le terme habituel du couplage spin-orbite, le terme en $\bar{l}^{2}$ a pour effet d'abaisser les états de grand moment angulaire. Les constantes $C$ ét $D$ ont été déterminées de façon à retrouver la suite des niveaux indiqués par Klinkenberg lorsque le facteur de déformation $\delta$ est nul. Nilsson obtient ainsi en fonction du facteur de déformation $\delta$, l'énergie des différents niveaux caractérisés par leur nombre quantique $\Omega$ et par leur parité, plusieurs niveaux peuvent avoir même 
valeur de $\Omega$ et même parité ; chaque niveau de $\Omega$ et de parité donnés est dû à la superposition avec des poids différents de contributions de fonctions d'ondes relatives à différents moments orbitaux ; par exemple un niveau $\Omega=3 / 2$ - correspond à des fonctions d'ondes de particules de moment orbital 5,3 et 1 .

Des comparaisons avec les résultats expérimentaux ont été faites par Mottelson et Nilsson [22] dans le cas de noyaux du groupe 1 ; pour obtenir un meilleur accord les auteurs ont été amenés à modifier légèrement les coefficients ajustables qui figurent dans l'hamiltonien (4) pour un nombre de protons compris entre 50 et 82 . Le facteur de déformation $\delta$ qui est assimilable au facteur $\beta$ de déformation de Bohr et Mottelson $(\delta \simeq 0,95 \beta)$ a été calculé pour chaque noyau à partir du moment quadrupolaire $Q_{0}$, qui est lui-même déterminé d'après les valeurs données par l'excitation coulombienne des noyaux.

Les spins et parités à l'état fondamental semblent

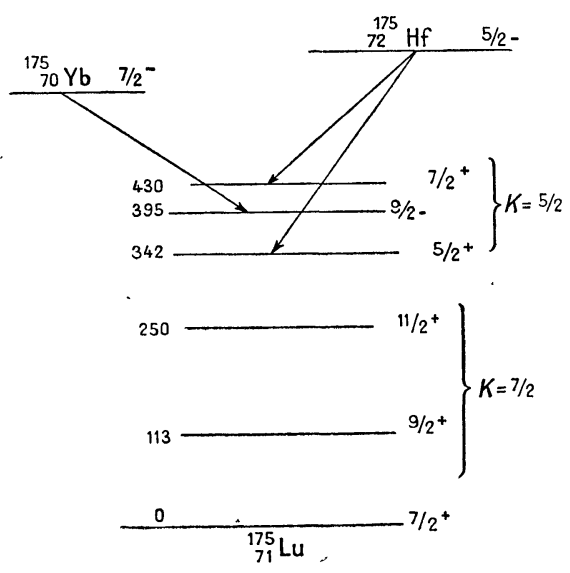

FIG. 4.

justifier les calculs de Nilsson ; ceux-ci facilitent aussi l'interprétation de certains états excités en particulier des états de spins voisins et de parités opposées entre lesquels ont lieu des transitions $\mathrm{E}_{1}$.

Nous prendrons comme exemples les niveaux de ${ }_{71}^{175} \mathrm{Lu}$ et ${ }_{63}^{153} \mathrm{Eu}$. Dans le premier [23], [24], [25], [26], [27][28] (fig. 4), l'état fondamental est $\Omega=7 / 2+$, les niveaux à 113 et $250 \mathrm{keV}$ mis en évidence par excitation coulombienne ainsi que dans les désintégrations de ${ }_{70}^{175} \mathrm{Yb}$ et ${ }_{72}^{175} \mathrm{Hf}$ pour le niveau de $113 \mathrm{keV}$, sont les niveaux de rotation du fondamental et ont pour spin $9 / 2$ et $11 / 2$ et la parité + . Le niveau à $395 \mathrm{keV}$ apparaît dans la désintégration ${ }_{70}^{175} \mathrm{Yb}$; d'après la valeur de $f t$ du spectre $\beta$ aboutissant à ce niveau, les corrélations angulaires entre les photons de 282 et $113 \mathrm{keV}$, les intensités relatives des photons de 395 et $282 \mathrm{keV}$ on peut attribuer au niveau de $395 \mathrm{keV}$ le caractère $9 / 2$. - Les niveaux de 342 et $430 \mathrm{keV}$ atteints lors de la désintégration de ${ }^{175} \mathrm{Hf}$ peuvent être considérés comme le fondamental et le premier état excité d'une bande de rotation $\Omega=K=5 / 2$, de parité + . D'après les courbes données par Mottelson et Nilsson on prévoit effectivement pour un noyau de numéro atomique $Z=71$ et de déformation $\delta=0,28$ des sous-couches voisines $\Omega=7 / 2+, \Omega=5 / 2+$ et $\Omega=9 / 2-{ }^{(1)}$.

Le cas de ${ }_{63}^{153} \mathrm{Eu}$ est plus complexe [27], [29], [30], [31] ( fig. 5), le fondamental est caractérisé par $\Omega=5 / 2+$. Les niveaux à 84 et $194 \mathrm{KeV}$ ont été obtenus par excitation coulombienne et aussi, avec une intensité très faible, dans la désintégration de ${ }_{62}^{153} \mathrm{Sm}$ pour le premier, ce sont les niveaux de rotation $7 / 2+, 9 / 2+d u$ fondamental. Les niveaux à 103 et $172 \mathrm{keV}$ apparaissent dans la désintégration de ${ }_{62}^{153} \mathrm{Sm}$ et ${ }_{64}^{153} \mathrm{Gd}$, on a été conduit à leur attribuer les caractéristiques $3 / 2+$ et $1 / 2+$.

Enfin dans la désintégration de ${ }^{153} \mathrm{Gd}$ apparaît le niveau à $98 \mathrm{keV}$ qui se désexcite par émission d'un photon très faiblement converti qui ne peut

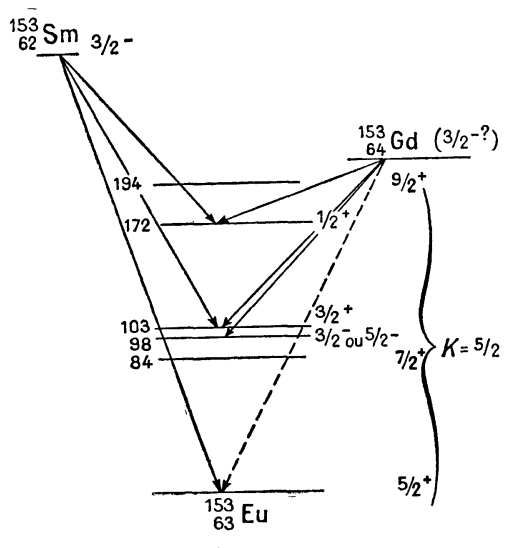

FIG. 5.

être qu'un dipôle électrique ; ce doit être un niveau $3 / 2$ - ou $5 / 2-$. Les courbes théoriques des énergies de niveaux font apparaître pour un nombre de protons voisin de 63 et unè déformation $\delta=0,3$ des niveaux successifs $5 / 2+, 3 / 2$, $1 / 2+$ : des niveaux $3 / 2$ - et $5 / 2$ - existant aussi dans ce domaine avec des énergies inférieures à celle du niveau $5 / 2$ + mais peu différentes.

$\mathrm{Si}$, comme nous venons de le voir, le modèle unifié permet d'expliquer un grand nombre des propriétés des niveaux excités des noyaux déformés, les schémas établis avec certitude sont encore peu nombreux. Bien des points restent à préciser et à comparer aux prévisions théoriques, en particulier les intensités relatives des diverses transitions et les périodes de ces états excités.

(1) Cette interprétation est en bon accord avec celle donnée récemment par Chase et Wilets (Phys. Res., 1956, 101, 1038). 


\section{BIBLIOGRAPHIE}

[1] Mayer (M. G.), Phys. Rev., 1949, 75, 1969.

[2] Haxel (O.), Jensen (J. H. D.), Suess (H. E.), $Z$. Physik, 150, 128, 295.

[3] Khinkenberg (P. F. A.), Rev. mod. Physics, 1952, 24, 63.

[4] Bонr (A.), Dan. Mat. Fys. Medd., 1952, 26, no 14.

[5] Bohr (A.) et Mottelson (B.), Dan. Mat. Fys. Medd., 1953, 27, no 16.

[6] Bон (A.), Rotational states of atomic nuclei, Munksjaards, 1954.

[7] ScharfF-Goldhaber (G.), Weneser (J.), Phys. Rev., 1955, 98, 212.

[8] Johansson (S. A. E.), Phys. Rev., 1955, 100, 835.

[9] Kerman (A. K.), En cours de publication à Dan. Mat. Fys. Medd.

[10] Murray (J. J.), Berm (F.), Marmer (P.), Du Mond (J. W. M.), Phys. Rev., 1955, 97, 1007.

[11] Stephens (Jr. F.), Asaro (F.), Perlman (I.), Phys. Rev., 1954, 96, 1568 ; Phys. Res., 1955, 100, 1543.

[12] Scharff-Goldhaber (G.), Der Mateosian (E.), Harbottle (G.), MaKeown (M.), Phys. Rev., 1955, $99,180$.

[13] Falk-Vairant (P.), Petit (G. Y.), C. R. Acad. Sc., 1955, 240, 296.

[14] Asaro (F.), Perlman (I.), Phys. Rev., 1954, 93, 1423.

[15] Perlman (I.), Stephens Jr. (F.), Phys. Res., 1955, 98, 262.
[16] Strominger (D.), Rasmussen (J. O.), Phys. Rev. 1955, 100, 844 .

[17] Engelikemeir (D.), Magnusson (L. B.), Phys. Rev. 1954, 94, 1395.

[18] Frilley (M.), Rosenblum (S.), Valadarès (M.), Bouissière (G.), J. Physique Rad., 1954, 15, 45.

[19] Milsted (J.), Rosenblum (S.), Valadarès (M.), $C . R$. Acad. Sc., 1954, 239, 700.

[20] Rasmussen Jr. (J. O.), Ark. Fys., 1953, Bd 7, N16.

[21] Nilsson (S. G.), Dan. Mat. Fys. Medd., 1955, 29, no16.

[22] Mottelson (B.R.), Nilsson (S. G.), Phys. Rev., 1955, 99, 1615.

[23] Marty (N.), C. R. Acad. Sc., 1955, 240,633.

[24] DE WAARD (H.), Phil. Mag., 1955, 46, 445.

[25] Akerlind, Hartman, Wieding, Phil. Mag., 1955, 46, 448.

[26] Mize (J. P.), Bunker (M. E.), Starner (J. W.), Phys. Rev., 1955, 100, 1390.

[27] Heydenburg (N. P.), Temmer (G. M.), Phys. Rev., $1955,100,150$.

[28] MaGlelland (M.), Mark (H.), Goodman (G.), Phys. Rev., 1955, 97, 1191.

[29] Bernstein (E. M.), Lewis (H. W.), B. A. P. S., 1956, vol. 1, 41 .

[30] Marty (N.), J. Physique Rad., 1955, 16, 458.

[31] Marty (N.), Vergnes (M.), C. R. Acad. Sc., 1956, 242, 1438 . 